\title{
CYSTOCRAN Tablets: A Natural Antibacterial Safeguard in UTI Infections
}

Research Article

Govind Shukla, Monica Yadav, Madugula Mahender \& C.J.Sampath Kumar

Lactonova Nutripharm (P) Ltd., Makers of CYSTOCRAN Tablet, 81/3 IDA Mallapur, Hyderabad, Telangana, India.

\section{Corresponding Author}

Author Email:

lactonovaresearch44@gmail.com

\section{INTRODUCTION}

\section{Urinary Tract Infection (UTI)}

A UTI is an infection in the urinary tract. Infections are caused by microbes-organisms too small to be seen without a microscope-including fungi, viruses, and bacteria. Bacteria are
Abstract: Urinary tract infections (UTIs) are a serious health problem affecting millions of people each year. Infections of the urinary tract are the second most common type of infection in the body. One woman in five develops a UTI during her lifetime. Natural remedies have been used for centuries to alleviate pain and discomfort as well as address the underlying symptoms. Natural remedies for urinary tract infections are safe and cause no side effects. They also support one's physical, mental, and emotional health, rather than simply treating UTI symptoms. A number of antibiotics can be prescribed to treat urinary tract infections. However, certain antibiotics may not be safe for these pregnant, plan to become pregnant or breast feeding women. Like all medications, antibiotics have side effects. These medications can produce reactions and even serious medical complications in some women. In addition, adverse reactions to antibiotics may be more serious in women with weakened immune systems, including women with diabetes. Possible side effects of antibiotics include allergic reactions, fever, nausea and bloating. Vaginal yeast infections are another common side effect of antibiotic use in women. Antibiotics kill bacteria, including bacteria that control the amount of other normal body organisms, such as yeast. So the reduction in bacteria can result in an overgrowth of yeast. Taking certain antibiotics during pregnancy may be a risky proposition since women who take them may deliver babies with increased risk of birth defects. The present Article reviews the role of cystocran tablets developed by R\&D cell of Lactonova Nutripharm Pvt Ltd. Hyderabad in Preventing Urinary tract infections.

Keywords: Urinary tract infections, Antibiotics, Symptoms.

the most common cause of UTIs. Normally, bacteria that enter the urinary tract are rapidly removed by the body before they cause symptoms. However, sometimes bacteria overcome the body's natural defenses and cause infection. An infection in the urethra is called urethritis. A bladder infection is called cystitis. Bacteria may travel up the ureters to multiply and infect the kidneys. A kidney infection is called pyelonephritis. The urinary tract is the body's drainage system for removing wastes and extra water. The urinary tract includes two kidneys, two ureters, a bladder, and a urethra. The kidneys are a pair of bean-shaped organs, each about the size of a fist and located below the ribs, one on each side of the spine, toward the middle of the back. Every minute, a person's kidneys filter about 3 ounces of blood, removing wastes and extra water. The wastes and extra water make up the 1 to 2 quarts of urine a person produces each day. The urine travels from the kidneys down two narrow tubes called the ureters. The urine is then stored in a balloon like organ called the bladder and emptied through the urethra, a tube at the bottom of the bladder. When the bladder empties, a muscle called the sphincter relaxes and urine flows out of the body through

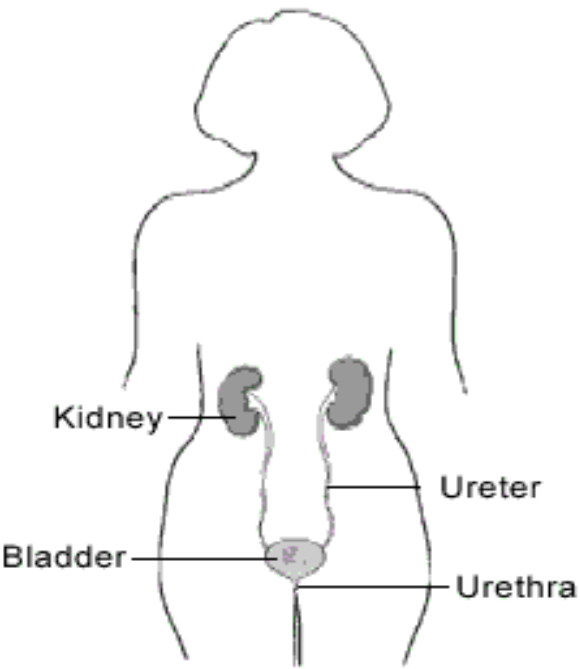

Fig.1 The urinary tract the urethra. The opening of the urethra is at the end of the penis in males and in front of the vagina in females. 
Most UTIs are caused by bacteria that live in the bowel. The bacterium Escherichia coli (E. coli) causes the vast majority of UTIs. Microbes called Chlamydia and Mycoplasma can infect the urethra and reproductive system but not the bladder. Chlamydia and Mycoplasma infections may be sexually transmitted and require treatment of sexual partners.

The urinary tract has several systems to prevent infection. The points where the ureters attach to the bladder act like one-way valves to prevent urine from backing up toward the kidneys, and urination washes microbes out of the body. In men, the prostate gland produces secretions that slow bacterial growth. In both sexes, immune defenses also prevent infection. But despite these safeguards, infections still occur. Certain bacteria have a strong ability to attach themselves to the lining of the urinary tract.

\section{UTIs in Women}

Urinary tract infections are the second most common type of infection in the body, accounting for about 8.1 million visits to health care providers each year. ${ }^{1}$ Women are especially prone to UTIs for anatomical reasons. One factor is that a woman's urethra is shorter, allowing bacteria quicker access to the bladder. Also, a woman's urethral opening is near sources of bacteria from the anus and vagina. For women, the lifetime risk of having a UTI is greater than 50 percent. ${ }^{2}$ UTIs in men are not as common as in women but can be serious when they occur.

\section{Risk factors for a UTI}

Although everyone has some risk, some people are more prone to getting UTIs than others. People with spinal cord injuries or other nerve damage around the bladder have difficulty emptying their bladder completely, allowing bacteria to grow in the urine that stays in the bladder. Anyone with an abnormality of the urinary tract that obstructs the flow of urine - a kidney stone or enlarged prostate, for example - is at risk for a UTI. People with diabetes or problems with the body's natural defense system are more likely to get UTIs.

Sexual activity can move microbes from the bowel or vaginal cavity to the urethral opening. If these microbes have special characteristics that allow them to live in the urinary tract, it is harder for the body to remove them quickly enough to prevent infection. Following sexual intercourse, most women have a significant number of bacteria in their urine, but the body normally clears them within 24 hours. However, some forms of birth control increase the risk of UTI. In some women, certain spermicides may irritate the skin, increasing the risk of bacteria invading surrounding tissues. Using a diaphragm may slow urinary flow and allow bacteria to multiply. Condom use is also associated with increased risk of UTIs, possibly because of the increased trauma that occurs to the vagina during sexual activity. Using spermicides with diaphragms and condoms can increase risk even further.

Another common source of infection is catheters, or tubes, placed in the urethra and bladder. Catheters interfere with the body's ability to clear microbes from the urinary tract. Bacteria travel through or around the catheter and establish a place where they can thrive within the bladder. A person who cannot urinate in the normal way or who is unconscious or critically ill often needs a catheter for more than a few days. The Infectious Diseases Society of America recommends using catheters for the shortest time possible to reduce the risk of a UTI. ${ }^{3}$ 
Many women suffer from frequent UTIs. About 20 percent of young women with a first UTI will have a recurrent infection. ${ }^{4}$ With each UTI, the risk that a woman will continue having recurrent UTIs increases. ${ }^{5}$ Some women have three or more UTIs a year. However, very few women will have frequent infections throughout their lives. More typically, a woman will have a period of 1 or 2 years with frequent infections, after which recurring infections cease. Men are less likely than women to have a first UTI. But once a man has a UTI, he is likely to have another because bacteria can hide deep inside prostate tissue. Anyone who has diabetes or a problem that makes it hard to urinate may have repeat infections. Research funded by the National Institutes of Health (NIH) suggests that one factor behind recurrent UTIs may be the ability of bacteria to attach to cells lining the urinary tract. One NIH-funded study found that bacteria formed a protective film on the inner lining of the bladder in mice. ${ }^{6}$ If a similar process can be demonstrated in humans, the discovery may lead to new treatments to prevent recurrent UTIs. Another line of research has indicated that women who are "nonsecretors" of certain blood group antigens may be more prone to recurrent UTIs because the cells lining the vagina and urethra may allow bacteria to attach more easily. A nonsecretor is a person with an $\mathrm{A}, \mathrm{B}$, or $\mathrm{AB}$ blood type who does not secrete the normal antigens for that blood type in bodily fluids, such as fluids that line the bladder wall. ${ }^{7}$

\section{Infections during Pregnancy}

Pregnant women seem no more prone to UTIs than other women. However, when a UTI does occur in a pregnant woman, it is more likely to travel to the kidneys. According to some reports, about 4 to 5 percent of pregnant women develop a UTI. ${ }^{8}$ Scientists think that hormonal changes and shifts in the position of the urinary tract during pregnancy make it easier for bacteria to travel up the ureters to the kidneys and cause infection. For this reason, health care providers routinely screen pregnant women for bacteria in the urine during the first 3 months of pregnancy.

\section{Signs and Symptoms of Urinary tract infections}

Symptoms of a UTI vary by age, gender, and whether a catheter is present. Among young women, UTI symptoms typically include a frequent and intense urge to urinate and a painful, burning feeling in the bladder or urethra during urination. The amount of urine may be very small. Older women and men are more likely to be tired, shaky, and weak and have muscle aches and abdominal pain. Urine may look cloudy, dark, or bloody or have a foul smell. In a person with a catheter, the only symptom may be fever that cannot be attributed to any other cause. Normally, UTIs do not cause fever if they are in the bladder. A fever may mean the infection has reached the kidneys or has penetrated the prostate. Other symptoms of a kidney infection include pain in the back or side below the ribs, nausea, and vomiting.

\section{Cystocran; A natural Safe Guard in Urinary tract infections.}

Urinary tract infections are a serious health problem affecting millions of people each year. Infections of the urinary tract are the second most common type of infection in the body. One woman in five develops a UTI during her lifetime. Natural remedies have been used for centuries to alleviate pain and discomfort as well as address the 
underlying symptoms. Natural remedies for urinary tract infections are safe and cause no side effects. They also support one's physical, mental, and emotional health, rather than simply treating UTI symptoms. A number of antibiotics can be prescribed to treat urinary tract infections. However, certain antibiotics may not be safe for these pregnant, plan to become pregnant or are breastfeeding women. Like all medications, antibiotics have side effects. These medications can produce reactions and even serious medical complications in some women. In addition, adverse reactions to antibiotics may be more serious in women with weakened immune systems, including women with diabetes. Possible side effects of antibiotics include allergic reactions, fever, nausea and bloating.

Vaginal yeast infections are another common side effect of antibiotic use in women. Antibiotics kill bacteria, including bacteria that control the amount of other normal body organisms, such as yeast. So the reduction in bacteria can result in an overgrowth of yeast. Taking certain antibiotics during pregnancy may be a risky proposition since women who take them may deliver babies with increased risk of birth defects.

Cystocran, A urinary antiseptic, that promotes overall health of urinary tract, gastrointestinal \& oral health Naturally without any side effect.

\section{Composition}

\section{Each tablet contains}

Cranberry Extract $200 \mathrm{mg}$

D.Mannose $200 \mathrm{mg}$

L.Acidophillus $150 \mathrm{mn}$

L.Salivarus $150 \mathrm{mn}$

L.Rhamnosus $150 \mathrm{mn}$

Fructooligo saccharides $50 \mathrm{mg}$

Vitamin A $5000 \mathrm{IU}$

Vitamin C $50 \mathrm{mg}$

Mechanism of Action of each ingredient of Cystocran tablet:

\section{Cranberry Extract}

Antibacterial Action, Inhibits bacterial adherence, Inhibits hemagglutination of E.coli.

Invitro studies have observed potent inhibition of bacterial adherence of E.coli and other gram-negative uro-pathogens

\section{D-Mannose}

- Prevents bacterial strains adherence

In vitro research has identified a mannose-specific lectin on the surface of adherent strains of E.coli 
Vitamin $C$

- Combats the growth of E.coli.

- Prevents bacterial infections in urinary tract.

- Repairs and maintains body tissues.

FOS

- Serves as a microflora substrate.

- Prevents yeast infections.

\section{L.acidophilus}

- Prevents uropathogenic bacterial adherence (J Infect Dis2001;183:485-491)

- Improves overall health.

- Effective against bacterial vaginosis

\section{L rhamnosus}

- Survives in the highly acidic conditions of GI tract

- Effective in antibiotic resistant Urinary Tract Infections of trimethoprim-sulfamethoxazole

- Safeguard the urogenital tract by excreting biosurfactants.

- Prevents adhesion of pathogens.

- Helps Build a Superior Immune System

- Stimulates the production of antibodies

- Combats dangerous invasive bacteria by phagocytosis.

\section{Vitamin A}

- Prevents urinary tract infections.

- Improves overall health.

- Helps to produce W.B.C.\& lymphocytes.

\section{Lactobacillus Salivarius}

- Shows Antimicrobial action.

- Eliminates harmful bacteria by Producing bacteriocins

- Effective against Salmonella dublin, E.coli, Candida albicans, Heliobacter pylori.

- Reduces inflammation. 
Other uses

- A single experimental study showed that the "high-molecular-weight constituent" of cranberry juice that inhibits the adherence of $E$. coli was effective in reversing and inhibiting the coaggregation of a large portion of dental plaque bacteria. Cranberry also has been recommended as an adjunctive treatment for Candida infections.

- In vitro studies have shown that cranberry juice exerts fungistatic effects against dermatophytic and other fungi but has no effect against Candida albicans. There are no controlled trials in humans evaluating the effectiveness of cranberry in treating fungal infections.

\section{Indications:}

Prevention and Arresting recurrent urinary tract infections in women

- Bacterial UTIs

- Interstitial Cystitis

\section{Dosage and Administration:}

- The recommended dosing for UTI prophylaxis is based on the most recent positive randomized controlled trial that used concentrated cranberry extract (200 to $400 \mathrm{mg}$ ) twice daily.

\section{Contraindications, Interactions, Adverse Effects}

- Cranberry has a record of safety, although specific long-term safety data are lacking. No significant herb-drug interactions have been reported.

- A single study found that cranberry may increase the absorption of vitamin $\mathrm{B}_{12}$ in patients who also are taking proton pump inhibitors and that it may allow the kidneys to metabolize weakly alkaline drugs (such as antidepressants and opioids) more rapidly, thus reducing their effectiveness.

- A small study found a significant rise in urinary oxalate levels, prompting a caution that regular use of cranberry may increase the risk of kidney stone formation in patients with a history of oxalate calculi.

\section{PHARMACOLOGICAL ACTION}

\section{Cranberry Extract in Cystocran}

Cranberries are a group of evergreen dwarf shrubs or trailing vines in the subgenus Oxycoccus of the genus Vaccinium. They can be found in acidic bogs throughout the cooler regions of the northern hemisphere.

Cranberry extract contains condensed tannins (or) proanthocyanidins (PACS) exhibiting microbial anti-adhesion properties by either of the following:

1. A-type PACs are metabolized relatively intact and collect in urine to provide protective effects from bacteria that migrate from the perineum and vagina. (or) 
2. The PACs eliminated through the colon bind to uropathogenic bacteria thus decreasing the virulence of the microbes if they come in contact with the uroepithelium.

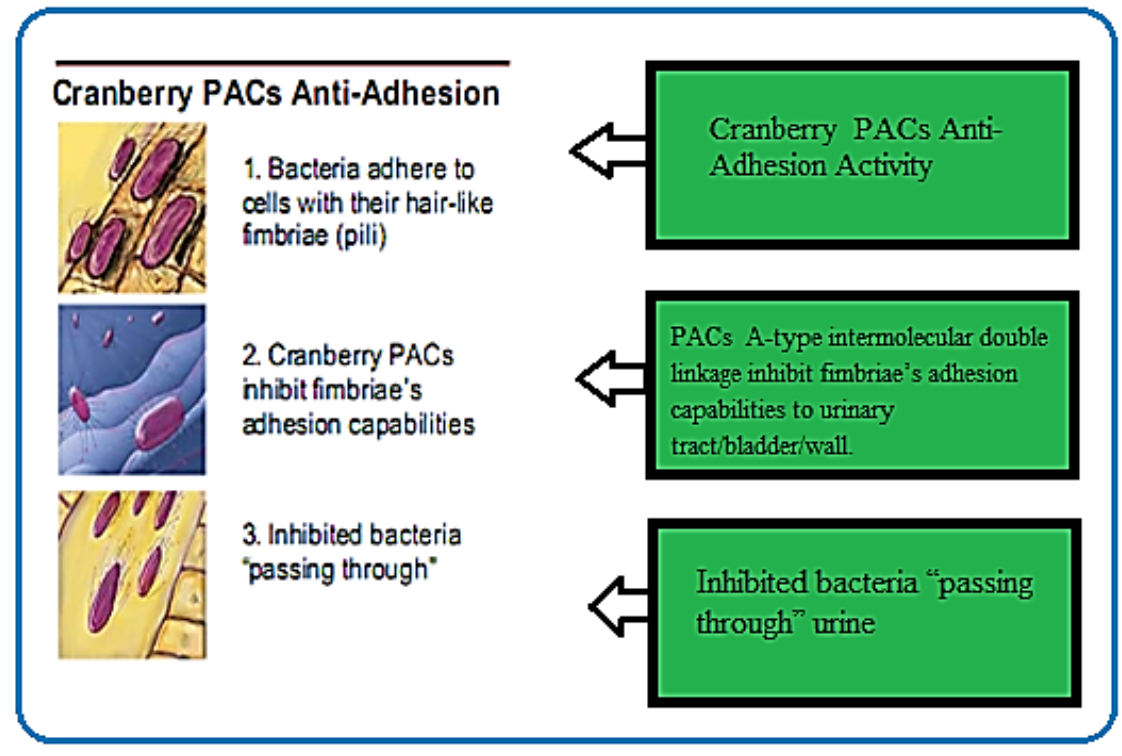

\section{Role of Cranberry in prevention of E coli infection:}

The E. coli bacteria cause $80-90 \%$ of urinary tract infections. Proanthocyanidins (PACs) from cranberries affect E. coli cells in three ways. Cranberry PACs (a). Change the shape of the E. coli from rods to spheres, (b). alter the cell membranes, and (c). Compress tendrils on the outside of the cells, which affects E. coli's ability to attach to cells lining the bladder wall.

\section{D-Mannose in Cystocran}

D-mannose is a simple sugar present in the cells that line the epithelial tract. It attaches to bacteria better than bacteria attaches to human cells in the bladder and urinary tract. D-mannose, is absorbed in the upper gastrointestinal tract and is transported into the blood stream largely unchanged. Therefore, a large amount of the sugar is extracted and added to the urine.
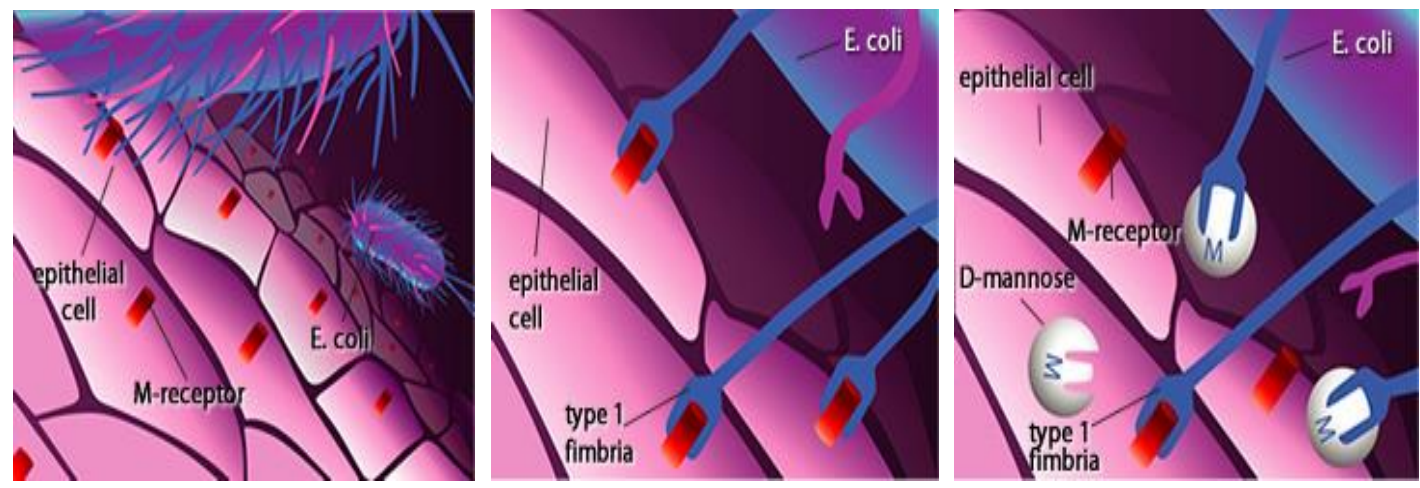
The "sugar-containing" urine will in turn coat any free-floating E. coli, and make it impossible for the bacteria to stick to the epithelial cells. Thus, the E. coli bacteria, which cause 90 percent of UTIs are rinsed out from the body upon normal urination. D-Mannose nourishes the healthy flora that colonise the membranes of the urinary tract and keep us in balance. D-Mannose does not interfere with normal blood sugar regulation.

\section{Pro \& Prebiotics in Cystocran}

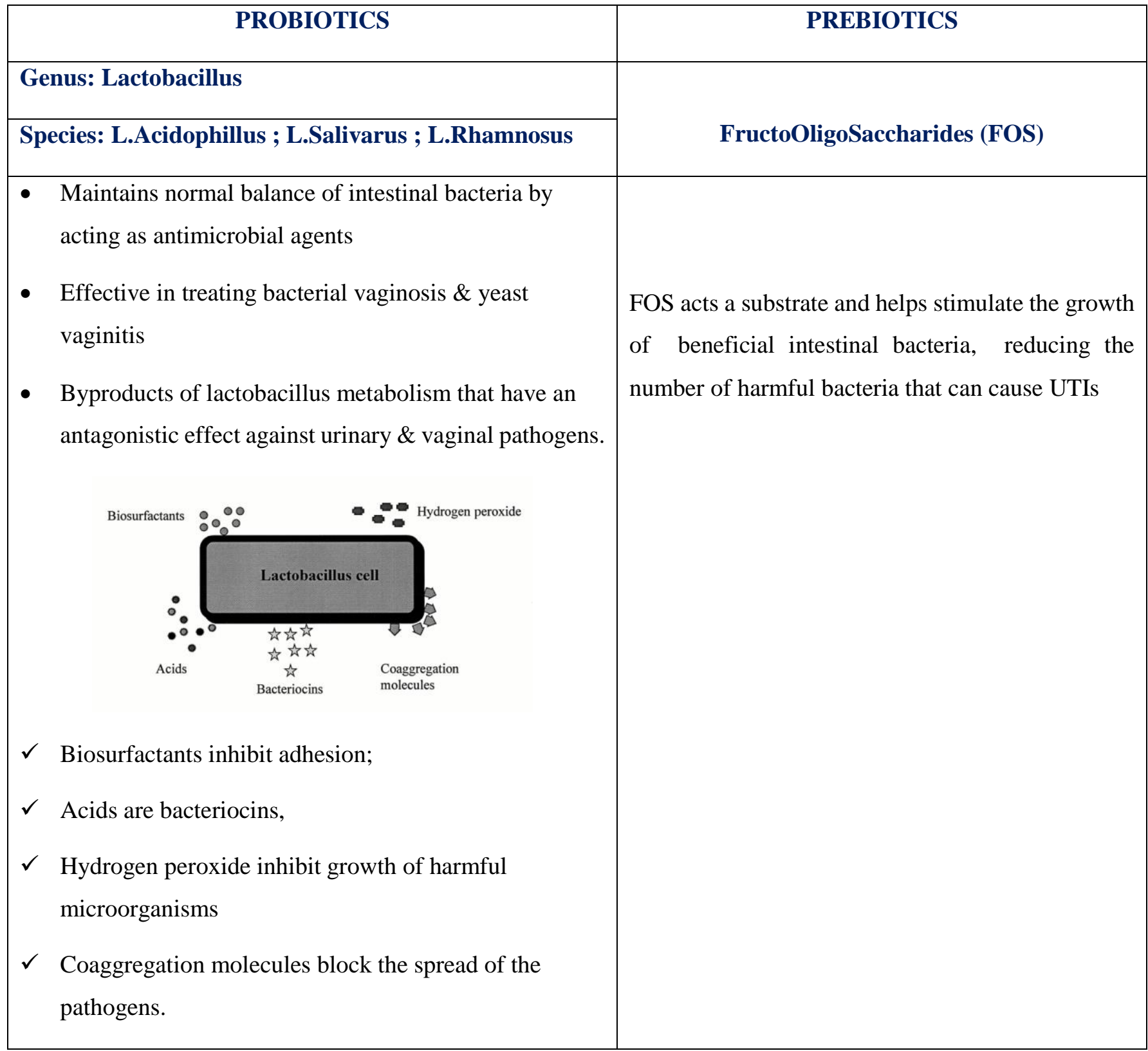

Vitamins in Cystocran

\begin{tabular}{|l|l|}
\hline \multicolumn{1}{|c|}{ VITAMIN A } & \multicolumn{1}{|c|}{ VITAMIN C } \\
\hline $\begin{array}{l}\text { Promotes healthy surface linings of urinary, \& } \\
\text { intestinal tracts }\end{array}$ & $* \begin{array}{l}\text { A powerful antioxidant that enhances immune } \\
\text { system and fights against bladder infection }\end{array}$ \\
& $*$\begin{tabular}{l} 
Helps decrease $\mathrm{pH}$, maintaining adequate \\
\hline
\end{tabular} \\
\hline
\end{tabular}


* Helps regulate the immune system, by destroying harmful microorganisms urine acidity, creating a less tolerable environment for bacteria to grow

Water and Vitamin $\mathrm{C}$ work in conjunction with cranberry extract to speed recovery from a UTI

\section{SUMMARY \& CONCLUSION}

Urinary tract infections are a serious health problem affecting millions of people each year. Infections of the urinary tract are the second most common type of infection in the body. One woman in five develops a UTI during her lifetime. Natural remedies have been used for centuries to alleviate pain and discomfort as well as address the underlying symptoms. Natural remedies for urinary tract infections are safe and cause no side effects. They also support one's physical, mental, and emotional health, rather than simply treating UTI symptoms. A number of antibiotics can be prescribed to treat urinary tract infections. However, certain antibiotics may not be safe for these pregnant, plan to become pregnant or are breast feeding women. Like all medications, antibiotics have side effects. these medications can produce reactions and even serious medical complications in some women. In addition, adverse reactions to antibiotics may be more serious in women with weakened immune systems, including women with diabetes. Possible side effects of antibiotics include allergic reactions, fever, nausea and bloating. Vaginal yeast infections are another common side effect of antibiotic use in women. Antibiotics kill bacteria, including bacteria that control the amount of other normal body organisms, such as yeast. So the reduction in bacteria can result in an overgrowth of yeast. Taking certain antibiotics during pregnancy may be a risky proposition since women who take them may deliver babies with increased risk of birth defects. Cystocran, A urinary antiseptic, that promotes overall health of urinary tract, gastrointestinal \& oral health Naturally without any side effect.

\section{REFERENCES}

1.Schappert SM, Rechtsteiner EA. Ambulatory medical care utilization estimates for 2006. National health statistics reports; no 8. Hyattsville, MD: National Center for Health Statistics; 2008.

2.Griebling TL. Urinary tract infection in women. In: Litwin MS, Saigal CS, eds. Urologic Diseases in America. Department of Health and Human Services, Public Health Service, National Institutes of Health, National Institute of Diabetes and Digestive and Kidney Diseases. Washington, D.C.: GPO; 2007. NIH publication 07-5512:587619.

3.Hooton TM, et al. Diagnosis, prevention, and treatment of catheter-associated urinary tract infection in adults: 2009 international clinical practice guidelines from the Infectious Diseases Society of America. Clinical Infectious Diseases. 2010;50(5):625-663.

4.Tolkoff-Rubin NE, Cotran RS, Rubin RH. Urinary tract infection, pyelonephritis, and reflux nephropathy. In: Brenner BM, ed. Brenner \& Rector's The Kidney. 8th ed. Vol. 2. Philadelphia: Saunders; 2008: 1203-1238. 
5.Schaeffer AJ. Infections of the urinary tract. In: Walsh PC, Retik AB, Vaughan ED, Wein AJ, eds. Campbell's Urology. 8th ed. Vol. 1. Philadelphia: Saunders; 2002: 515-602.

6Anderson GG, Palermo JJ, Schilling JD, et al. Intracellular bacterial biofilm-like pods in urinary tract infections. Science. 2003;301:105-107.

7.Stapleton AE, Nudelman E, Clausen H, Hakomori S, Stamm WE. Binding of uropathogenic Escherichia coli R45 to glycolipids extracted from vaginal epithelial cells is dependent on histo-blood group secretor status. Journal of Clinical Investigation. 1992;90;965-972.

8.Sharma JB, Aggarwal S, Singhal S, Kumar S, Roy KK. Prevalence of urinary incontinence and other urological problems during pregnancy: a questionnaire based study. Archives of Gynecology and Obstetrics. 2009;279(6):845-851. 